\title{
LAS RELACIONES ENTRE COREA DEL NORTE Y PAKISTÁN Y EL TRÁFICO DE TECNOLOGÍA
}

\author{
Gracia Abad ${ }^{1}$ \\ Universidad de Nebrija/UNISCI
}

\begin{abstract}
Resumen:
Corea del Norte supone una amenaza para la paz y seguridad internacionales no sólo como consecuencia de sus programas de armas nucleares y de misiles sino también y sobre todo de la transferencia de tales ingenios. En el marco de tal transferencia la relación del estado del nordeste de Asia con Pakistán merece ser objeto de especial atención. El artículo, en ese sentido, analiza los intercambios en materia nuclear y de misiles entre Pakistán y Corea del Norte y la situación a corto-medio plazo en relación con las transferencias norcoreanas.
\end{abstract}

Palabras clave: Corea del Norte, Pakistán, nuclear, armas, misiles, Khan.

Title in English: "North Korea-Pakistan Relations and Technology Transfers".

\begin{abstract}
:
North Korea may well constitute a threat for international peace and security not only for its nuclear weapons and missile programs but also due to the transfer of such know-how. In this regard, the relation between the North Korean State and Pakistan deserves special attention. Thus, the article will analyze the nuclear and missile exchanges between Pakistan and North Korea and the short, medium term implications of the North Korean transfers.
\end{abstract}

Keywords: North Korea, Pakistan, nuclear, weapons, missiles, Khan.

Copyright (C) UNISCI, 2012.

Las opiniones expresadas en estos artículos son propias de sus autores, y no reflejan necesariamente la opinión de UNISCI. The views expressed in these articles are those of the authors, and do not necessarily reflect the views of UNISCI.

\footnotetext{
${ }^{1}$ La Dr. Gracia Abad es profesora de Relaciones Internacionales en la Universidad Nebrija en Madrid y es Investigadora Senior en UNISCI, Universidad Complutense.

E-mail: graciaabad@yahoo.es.

http://dx.doi.org/10.5209/rev_UNIS.2012.n29.406ロ
} 


\section{Introducción}

Las recurrentes noticias acerca de los programas de armas nucleares de Corea del Norte hacen que tengamos bien presente el problema que constituye el estado asiático en términos de proliferación vertical. Sin embargo, quizás no seamos siempre igual de conscientes de las implicaciones del proceder del gobierno de Pyongyang en términos de proliferación horizontal. Y es que, más allá de la amenaza que pueda suponer -especialmente para sus vecinos- que Corea del Norte cuente cada vez con más y mejores sistemas de armas nucleares, en términos globales, el tráfico de tecnología y secretos nucleares, pero también de misiles por parte del estado ermitaño ha sido, es y puede seguir siendo, si cabe, más preocupante.

En este sentido no parece falto de sentido pensar que para Corea del Norte la transferencia de tecnología y know how sea al menos tan importante como en el propio desarrollo de armas nucleares ya que, junto a consideraciones de seguridad y orgullo nacional, habría que mencionar muy probablemente razones de carácter económico entre los motivos que impulsan a la República Popular Democrática de Corea a seguir con sus programas nuclear, de armas nucleares y de misiles.

$\mathrm{Y}$ es que, mientras cuente con el producto, sólo necesita buscar potenciales compradores.

\section{Las relaciones militares con Pakistán}

En principio no habría ningún elemento que hiciera sospechar que las relaciones entre Corea del Norte y Pakistán pudieran ser importantes; se trata de dos estados distantes, pertenecientes a dos subregiones de Asia con situaciones diferentes y, si se quiere, en esa misma línea, miembros de contextos de seguridad regional también distintos.

\subsection{Los intercambios}

Sin embargo, en la búsqueda de intercambios comerciales en materia de armamento entre los dos Estados nos podemos remontar más de tres décadas atrás, a 1971, cuando, en el contexto de la Guerra Indo-Pakistaní Corea del Norte se revela como suministradora -aunque modestade armas convencionales a Pakistán ${ }^{2}$.

Posteriormente, desde finales de los 80 y comienzos de los noventa la relación va a cobrar mucha más fuerza. Así ambos estados trabajarán conjuntamente en el desarrollo del programa de misiles iraní y Pakistán serviría de intermediario entre Corea del Norte por una parte e Irán y Libia por otra. Del mismo modo, Pakistán serviría a Corea del Norte como intermediario en el suministro de armas de ésta a Irán en el contexto de la guerra irano-iraquí, suministro que ocasionaría la imposición de sanciones a Corea del Norte.

Para entonces estos Estados tienen en común dos cosas: su voluntad de adquirir tecnología para la fabricación de armamentos y su falta de liquidez. No tardarían en llegar a la conclusión de que sus deseos eran en realidad complementarios, pues Pakistán estaba interesado en tecnología de misiles, algo con lo que Corea del Norte contaba mientras que ésta quería que le proporcionaran centrifugadoras, planos y tecnología de enriquecimiento de

\footnotetext{
${ }^{2}$ Panda, Rajaram: "India-Republic of Korea Military Diplomacy: Past and Future Projections" Perspectives, Journal of Defence Studies, vol. 5, no. 1 (January 2011), p. 20.
} 
uranio para poner en marcha un programa de armas nucleares a partir del uranio enriquecido que sería, a la sazón, el nudo gordiano del conflicto nuclear con Corea del Norte y Pakistán los poseía.

En función de ese acuerdo, Pakistán puso en marcha dos programas de misiles:

Un primer programa en el que también recibiría asistencia tanto de China como de algún país europeo. Los resultados de ese programa no se harían esperar: Pakistán comenzaría desarrollando un misil de corto alcance, el Haft $1^{3}$ que, además, era demasiado pequeño para poder equiparlo con cabezas nucleares, al que seguiría pronto el Haft 2 (Abdali) y el Haft 3 (Ghaznavi) de alcance intermedio (con alcance de hasta $300 \mathrm{~km}$ frente a los $80 \mathrm{~km}$ del anterior y que, en realidad, constituían desarrollos de M-9 chino. El paso siguiente sería el Haft 4 (Shaheen 1) un misil de 300-600 km de alcance mucho más evolucionado ya que estaba basado en el M-11 chino. A él seguiría el Haft 5 en su versión Ghauri 1 con un alcance de 1300 Finalmente y, también sobre la base de modelos chinos, desarrolló el Haft 6 (Shaheen2), un misil intercontinental, con alcance entre 2000 y $2300 \mathrm{~km}$. Entre los misiles mencionados, el Haft 3, el Haft 4 (Shaheen 1) y el Haft 5 (Ghauri 1) pueden ser equipados con cabezas nucleares ${ }^{4}$.

Un segundo programa, en el que Corea del Norte también le presta apoyo, tenía la finalidad de desarrollar misiles similares a los Scud soviéticos. La clave de este programa estaría en los laboratorios del ya famoso doctor Khan en Kahuta. En efecto, 1992 y 1993 científicos pakistaníes habrían realizado sendos viajes para conocer las instalaciones norcoreanas y los misiles No Dong y asistir a una demostración de vuelo de un No Dong. Se ha llegado a afirmar que la entonces Primera Ministra Benazir Bhutto visitó personalmente Pyongyang para supervisar la compra de misiles ${ }^{5}$ aunque es algo que no está comprobado y que la propia Primera Ministra Bhutto nunca reconoció ${ }^{6}$, a pesar de que se afirma incluso que en el viaje de vuelta llevaba con ella un misil desensamblado ${ }^{7}$. El resultado de aquellos acuerdos sería, en cualquier caso, la compra en metálico de algunos misiles No Dong.

Lejos de cesar, los intercambios se irían intensificando y, en 1994, se firmaría un acuerdo tripartito entre Pakistán, China y Corea del Norte orientado a poner las bases para la cooperación en cuestiones técnicas.

Entre las consecuencias de esos acuerdos hay que situar probablemente el envío de más de una decena de misiles NoDong desensamblados de Corea del Norte a Pakistán en el curso de los cuatro años siguientes. Asimismo, en los años siguientes Pakistán comenzó el desarrollo de nuevos misiles, el Ghauri III y el Tipu, que no eran sino una evolución de los Taepo Dong I y II desarrollados por la República Popular Democrática de Corea. Del primero se esperaba que contara con un alcance de 2500-3000 km, mientras que el segundo se pensaba que podría llegar a tener incluso un alcance de $4000 \mathrm{~km}$. Para Pakistán estos desarrollos tenían especial importancia dada su carrera armamentística -y su conflicto- con la India, que

\footnotetext{
3 Abad Quintanal, Gracia (2007): “Asia: Una región Inestable”, en Zeraoui, Zidane: La Paz y las Regiones, Monterrey, Fondo Editorial Nueva León, p. 65.

${ }^{4}$ Garrido, Vicente: "Pakistán, armas nucleares y seguridad”, Política Exterior, no121 (Febrero-Marzo 2008), p. 116.

${ }^{5}$ Levy, Adrian y Scott-Clark, Catherine (2007): Deception. Pakistan, the US and the Secret Trade in Nuclear Weapons, London Atlantic Books, p. 245.

${ }^{6}$ También formaron parte de la delegación un grupo de generales entre los se contaban Khawaja Ziauddin y Pervez Musharraf.

${ }^{7}$ Bagchi, Indrani “Kim Jong-Il's Death: India fears revival of North Korea-Pakistan nuclear bonding”, The

Times of India, 20 de diciembre de 2011, en http://articles.timesofindia.indiatimes.com.
} 
acababa de desarrollar, los Agni II y III, el último de los cuales se consideraba que podía alcanzar los $3500 \mathrm{~km}^{8}$

Al propio tiempo, Corea del Norte estaría recibiendo de Pakistán modelos de prototipos de centrifugadoras de gas de alta velocidad y planos para su construcción. En efecto, como también ocurriera con Irán o Libia, todo parece indicar que la República Popular Democrática de Corea trataba de hacerse con las centrifugadoras P-1 de los laboratorios (KRL), dirigidos por el doctor Khan, en lo que constituye el que quizás es el elemento más famoso de cooperación técnica entre Corea del Norte y Pakistán. Tal interés se explica porque las centrifugadoras eran la pieza clave que permitiría a Corea del Norte el desarrollo de un programa nuclear a partir del enriquecimiento de uranio, procedimiento mucho más difícil de detectar ${ }^{9}$.

Sería ya en la segunda mitad de la década de los noventa cuando la cooperación con los laboratorios KRL se hiciera particularmente intensa. Con todo, van a ser las motivaciones de carácter económico las que finalmente harán que Pakistán transfiera las centrifugadoras, y es que no encontró otro modo de pagar la deuda de cuarenta millones de dólares contraída como consecuencia de la adquisición de los misiles tipo NoDong efectuada en 1993.

Más allá de todo ello, los intercambios de visitas que permitieran sustanciar esa cooperación fueron constantes en esos años. Así, los viajes de científicos pakistaníes e, incluso, de la propia Primera Ministra Benazir Bhutto a Pyongyang a principios de los noventa fueron sólo el comienzo de lo que se calcula que llegó a ser casi una decena de viajes mensuales en ambos sentidos entre Corea del Norte y Pakistán.

En dichos viajes participan, por ejemplo, por parte pakistaní, entre otros, el propio Dr. Khan, que visitaría Corea del Norte hasta en 13 ocasiones, o los generales Ziauddin y Sajawal $^{10}$.

Del mismo modo, por parte norcoreana viajarán en distintos momentos a Pakistán Pak Cheng-kuk, vicepresidente de la Asamblea Popular de Corea del Norte, que visitó tanto Pakistán como Irán junto con un grupo de personalidades dedicadas al programa nuclear; Choe Hui-chong, presidente de la Comisión de Ciencia y Tecnología, que visitó Pakistán como cabeza de un grupo de expertos en tecnología de misiles; o Choe Kwang, Vicepresidente de la Comisión de Defensa, Ministro de las fuerzas armadas y jefe del ejército que acudió a Islamabad con el fin de firmar un acuerdo por el que Corea del Norte suministraba misiles No-Dong, motores y tanques de combustible líquido.

Hay que añadir, en este sentido, que un grupo de coreanos llegaría incluso a instalarse en los laboratorios KRL, empleando para ello a la empresa coreana Changgwang Sinyong Corporation como tapadera, para asesorar a Pakistán en su programa de misiles y que, de hecho, a la altura de 1998, científicos norcoreanos asistirían a Pakistán en la prueba de una versión modificada del NoDong.

\footnotetext{
${ }^{8}$ Abad Quintanal, op. cit. p. 65.

9 Abad, Gracia y Priego, Alberto: "Inteligencia y Contraproliferación. Las Relaciones entre Corea del Norte y Pakistán”, Inteligencia y seguridad: Revista de Análisis y Prospectiva, no 4 Julio (Noviembre de 2008), p. 34.

${ }^{10}$ Squassoni, Sharon A.: "Trade between North Korea and Pakistan" CRS Report for the Congress, 28 de noviembre de 2006, p. 110.
} 
En los años siguientes, como decíamos, Pakistán y Corea del Norte seguirían intercambiando misiles por tecnología nuclear ${ }^{11}$ algo que a la altura de 2003 haría que EEUU impusiera sanciones a Corea del Norte por la venta clandestina de misiles.

Con el tiempo, la transferencia de sistemas para desarrollar un programa nuclear de uranio enriquecido sería crecientemente conocida e, incluso, reconocida por el propio Dr. Khan que, cuando menos, aceptaba haber proporcionado a Corea del Norte planos de centrifugadoras y prototipos ${ }^{12}$.

Ahora bien, ese conocimiento llevaría a la certeza acerca de la existencia en Corea de tal programa, extremo negado hasta fecha relativamente reciente por el régimen de Pyongyang ${ }^{13}$.

Estos descubrimientos supondrán, por otra parte, el fin de la red de tráfico nuclear que lideraba el Dr. Khan y en la que, junto a los dos Estados que nos ocupan, participaban Estados como Irán, Egipto o Libia como hemos apuntado más arriba (Musharraf 2006), pero no necesariamente al fin de la cooperación militar entre Pakistán y Corea del Norte.

\subsection{La red del Dr. Khan y las acusaciones de corrupción}

Sin embargo, tales hallazgos no permitirían conocer con detalle todos los elementos de la ya larga relación entre Pakistán y Corea del Norte. De hecho, hace sólo unos meses, de la mano del estadounidense Washington Post ha salido a la luz que Pyongyang habría incluso pagado sobornos a altos mandos militares pakistaníes como parte de los acuerdos hechos a mitad de los noventa. En efecto, el Dr. Khan habría filtrado a dicho diario una copia de una carta supuestamente enviada en 1998 por un alto funcionario norcoreano en la que se afirma que Corea del Norte pagaría 3 millones de dólares a Jehangir Karamat, antiguo jefe militar pakistaní mientras que otro general pakistaní habría sido el destinatario de medio millón de dólares además de tres conjuntos de diamantes y rubíes ${ }^{14}$. Jehangir Karamat, por su parte, no ha dudado en afirmar la carta es falsa, algo en lo que también insisten algunos otros altos funcionarios pakistaníes ${ }^{15}$.

La carta, fechada el 15 de julio de 1998, firmada supuestamente por el Secretario del Partido de los Trabajadores de Corea del Norte y marcada como "secreta", de ser auténtica, serviría para probar que el Dr. Khan no actuó de forma autónoma como se ha dicho en alguna ocasión, sino que lo hacía con respaldo oficial, pero también pondría de manifiesto el tremendo interés norcoreano en la relación. En ese mismo sentido, también confirmaría que Corea del Norte estaba tratando de poner en marcha un programa nuclear basado en uranio enriquecido mientras se comprometía a abandonar el de plutonio que ya llevaba en marcha algún tiempo ${ }^{16}$.

\footnotetext{
11 "Pakistan-North Korea Weapons Trade Continued Through 2002", Pakistan Defence, en www.defence.pk.

${ }^{12}$ Bechtol, Bruce E. (2007): Red Rogue: the Persistent Challenge of North Korea, Virginia, Potomac Books, p. 17.

13 Smith, Jeffrey: "Pakistan's nuclear-bomb maker says North Korea paid bribes for know-how", The Washington Post, 7 de Julio de 2011, en

http://www.washingtonpost.com/world/national-security/pakistans-nuclear-bomb-maker-says-north-korea-paidbribes-for-know-how/2010/11/12/gIQAZ1kH1H_story.html.

${ }^{14}$ McNaughton, Graeme: "Khan: Pakistan sold nuclear weapons technology to North Korea", Digital Journal, 7 de Julio de 2011, en http://digitaljournal.com/print(article/308876).

${ }^{1515}$ Smith, op. cit.

${ }^{16}$ Rozen, Laura: “AQ Khan: North Korea bribed Pakistani generals for nuclear technology”, Yahoo News, 7 de Julio de 2011.
} 
En ella se pedía supuestamente que se entregara al funcionario norcoreano pertinente en la embajada en Pakistán los documentos y componentes acordados de forma que puedan ser enviados a Pyongyang cuando el avión fuera de vuelta a Corea del Norte después de la entrega de los componentes de misiles ${ }^{17}$.

Determinar la autenticidad de la carta parece difícil pues, mientras algunas fuentes oficiales estadounidenses afirman que la firma parece auténtica, es similar a otras cartas norcoreanas, el contenido de la carta coincide con la información que se tiene hasta el momento acerca de la cooperación entre Corea del Norte y Pakistán y la información que aparece en la carta era conocida por muy pocos; tampoco faltan quienes indican que las cartas norcoreanas de esa época aparecen fechadas según el calendario Juche y no según el occidental como ocurre en la carta entregada por Khan. Asimismo, la carta, escrita en inglés, daría la impresión de estar redactada por alguien cuya lengua nativa era el inglés, pero es oriundo de Asia meridional dada la peculiar utilización de algunos tiempos verbales ${ }^{18}$.

Con todo, no deja de ser interesante que en la carta se mencione a Mr. Jon, funcionario norcoreano que habría estado destinado también en Siria y Libia, Estados con los que Corea del Norte ha traficado secretos nucleares.

Asimismo, el supuesto autor de la carta sería Jon Byong Ho, quien, según fuentes de inteligencia de Estados Unidos, habría sido el encargado en Corea del norte del comercio de tecnología y misiles ${ }^{19}$.

\section{China: el avalista}

Con todo, una parte importante de la cooperación entre Corea del Norte y Pakistán no habría sido posible sin el apoyo chino.

En efecto, comentábamos más arriba cómo para mediados de los noventa se firmó un acuerdo tripartito entre China, Corea del Norte y Pakistán que serviría de base para la cooperación técnica entre los tres países. En efecto, el acuerdo tenía sentido en la medida en que permitía que Corea del Norte asistiera a Pakistán en el desarrollo de misiles y lo hiciera contando con tecnología y sistemas de guiado que previamente le había suministrado la República Popular China que, sin embargo, salvaba así las apariencias en lo que al cumplimiento del MCTR respecta, ya que no transfería tecnología directamente a Pakistán ${ }^{20}$. Pues bien, dicho acuerdo parece estar en consonancia con una dinámica que se seguiría acentuando en los años siguientes pero que tampoco era nueva. De hecho, el comercio de armas entre Corea del Norte e Irán en el que, como comentábamos antes Pakistán actuaba como intermediario, siempre contó con el visto bueno de Beijing.

Y es que, si por una parte la República Popular China es casi el único aliado con que cuenta Pyongyang, las relaciones entre Islamabad y Beijing, sin haber sido nunca malas,

\footnotetext{
17 "North Korea paid Pakistan for Nuclear Weapons tech", Associated Press, 2 de Julio de 2011, en www.cbc.ca/news/world/story/2011/07/07/nuclear-pakistan-nkorea.html.

${ }^{18}$ Farrell, Tad: “Did North Korea really bribe Pakistan?”, NK News, Julio de 2007, en http://www.nknews.org/2011/07/did-north-korea-really-bribe-pakistan/.

${ }^{19}$ McNaughton, op. cit.

${ }^{20}$ Panda, op. cit., p. 21 Ver también Tkacik, John K.: “The enemy of Hegemony is My Fiend: Pakistan's de facto 'Alliance' with China”, House Committee on Foreign Affairs Oversight and Investigations Subcommittee, 26 de Julio de 2011, p. 7.
} 
parecen ser cada vez mejores. Esto último parece particularmente comprensible si tenemos en cuenta no sólo el progresivo distanciamiento entre Pakistán y Estados Unidos, consecuencia en buena medida de la Guerra contra el Terrorismo, sino de la creciente sintonía entre Estados Unidos y la India. La República Popular China, que ve a la India como un rival cuando menos a nivel regional, ve en el estrechamiento de la relación con Pakistán un medio para reequilibrar la situación.

\section{Expectativas a corto-medio plazo}

Ahora bien, como decíamos el fin de la red de Khan no tiene por qué suponer necesariamente el de la colaboración entre Corea del Norte y Pakistán que, en cambio, podría seguir activa pese a que sus protagonistas insistan en negarlo.

Por otra parte, si tenemos en cuenta que Pakistán es considerado como uno de estados que, potencialmente, albergan mayor número de elementos terroristas ${ }^{21}$, la continuidad de los intercambios con Corea del Norte, ya sean oficiales ya tengan como destino a esos grupos terroristas, parece tan lógica como preocupante para la sociedad internacional aunque, eso sí, lucrativa para la República Popular Democrática de Corea.

En ese mismo sentido, como ya ocurriera en el pasado y se ha apuntado más arriba, los tráficos norcoreanos también podrían tener como destinatarios Estados distintos de Pakistán, pero deseosos de adquirir armas nucleares o capacidad para fabricarlas o bien a los grupos terroristas que se escondan en ellos.

En consecuencia, no hay ninguna razón para pensar que a corto-medio plazo Corea del Norte deje de ser un agente de proliferación horizontal, renunciando con ello a unos ingresos que bien pueden contribuir a la subsistencia del peculiar régimen de Pyongyang.

\footnotetext{
${ }^{21}$ Thakur, Ramesh: "Pakistan and North Korea live on the Edge", The Globe and Mail, 1 de diciembre de 2010, en http://www.theglobeandmail.com/news/opinions/opinion/pakistan-and-north-korea-live-on-theedge/article1819651/.
} 